\title{
Síndrome de abstinencia y otros efectos en jóvenes fumadores de hachís
}

\author{
Ma Dolores Baño Rodrigo*, José Antonio Ramos Atance**, Ma Luz López Garcia***, \\ Valentín de Antonio Pérez****, José Luis Guillén Llera***** \\ * Psiquiatra-Coordinadora. Programa Municipal de Drogas. Majadahonda. Madrid \\ **Catedrático de Universidad. Departamento de Bioquímica. Facultad de Medicina (UCM) \\ ***Doctor en Farmacia. Programa Municipal de Drogas. Majadahonda \\ **** Educador social. Programa Municipal de Drogas. Majadahonda \\ ***** ProfesorTitular Universidad. Escuela de análisis clínicos. Facultad de Farmacia (UCM) \\ Enviar correspondencia a: \\ Ma Dolores Baño. C/ Doctor Calero n² $203^{\text {a }}$ planta. ·fno: $916387090 \cdot 28220$ Majadahonda · Email: lolabr@infonegocio.com
}

\section{RESUMEN}

Este estudio evalúa los efectos derivados del consumo de hachís y la aparición de síntomas de abstinencia en 145 jóvenes que acuden a un programa de tratamiento de Mayo a Octubre de 2002 para evitar el pago de la sanción administrativa. Todos ellos realizan un cuestionario anónimo, con el fin de evaluar el consumo de otras drogas, los efectos producidos por el consumo de hachís, diferenciando dos tipos de consumidores (diario y esporádico o fines de semana) y su relación con la aparición de síntomas de abstinencia y complicaciones derivadas.

Los resultados obtenidos, muestran un perfil de jóvenes policonsumidores de hachís, alcohol y tabaco que consumen con amigos la gran mayoría. Confirma la aparición de síntomas de abstinencia, como aumento del consumo de tabaco, alteraciones del apetito y sueño, nerviosismo y síntomas depresivos. Los consumidores habituales tuvieron además, más deseos de volver a consumir e inquietud. Este último grupo consumió más en situaciones de peligro tales como conducir y mostraron mas síntomas de apatía. No queda claro la dosis necesaria que determinó la aparición de esta sintomatología ni si existieron factores de vulnerabilidad individual.

Palabras Clave: cannabis, jóvenes, consumo diario o fines de semana, síntomas de abstinencia.

\section{SUMMARY}

This study examines the effects derived from hashish use and the appearance of abstinence symptoms in a population of 145 young users who were in a treatment programme from May to October 2002 in order to avoid paying a fine imposed by the courts. Differentiating two kinds of user (daily and sporadic or at weekends only), they all answered an anonymous questionnaire to evaluate the frequency of their use of other drugs, the effects caused by hashish smoking, and the relationship with the appearance of withdrawal symptoms and subsequent secondary effects.

The results show a pattern of the multiple use of hashish, alcohol and tobacco by young users, the majority with their friends. They also confirm the appearance of abstinence symptoms such as a rise in tobacco consumption, alterations in sleep and appetite, irritability and depressive symptoms. Moreover, the daily users had greater cravings to smoke hashish and were more restless. The rate of consumption in this last group was even higher in dangerous situations such as driving a car, and they also showed more symptoms of apathy. The doses of hashish necessary to cause withdrawal symptomatology, were not clear, however, and nor was the existence of individual vulnerability factors.

Key Words. Cannabis, young, people, daily and weekend users, withdrawal symptoms.

\section{INTRODUCCIÓN}

$\mathbf{F}$ umar hachís es la práctica de consumo ilegal más habitual en Europa y Estados Unidos. Su uso aumenta progresivamente desde 1990 (Substance Abuse and mental Health Services Administration 1999). Se observa una tendencia al policonsumo de cannabis, alcohol y tabaco en muchos países, entre ellos España, según reflejan los datos del Plan Nacional sobre drogas (PNSD, 2000), cambiando el tipo de policonsumo en otros países como Alemania, donde los resultados de un estudio realizado con una muestra de 3201 jóvenes de 14 a 24 años, mostraron que el cannabis era la droga mas utilizada, pero el patrón de policonsumo era con estimulantes y alucinógenos fundamentalmente (Perkonnig et al. 1999). 
La cuantificación de las dosis administradas y la relación dosis-efecto en un cierto rango, es una condición básica para establecer el criterio de actividad farmacológica. En el caso del cannabis, el estudio de dicha relación, aun sabiendo que el principio activo es el delta- 9- tetrahidrocannabinol (THC), que actúa sobre receptores CB1, no se tiene hasta el momento un conocimiento exhaustivo sobre el tema (Meana J y Ulibarri I. 1999).

Por otra parte, el síndrome de abstinencia a cannabis no está incluido en el DSM IV (American Psychiatric Association 1994) estando todavía sin esclarecer su significado clínico.

El hachís no presenta un síndrome de abstinencia tan definido como los opiáceos (Smith NT, 2002). Algunos estudios clínicos realizados en consumidores de altas dosis de cannabis indican que el cese del consumo no produce un síndrome de abstinencia con sintomatología severa (Abood y Martin 1992; Haney et al, 1999a, 1999b). Esta observación clínica coincide con algunos resultados obtenidos en animales de experimentación, en los que no se ha podido observar en las diferentes especies animales estudiadas, la aparición de manifestaciones somáticas de un síndrome de abstinencia espontáneo tras el cese del tratamiento crónico con THC, ni siquiera tras la administración de dosis sumamente elevadas (Budney et al 2000, Aceto et al 2001), siendo necesarios para la aparición de los síntomas la administración de SR141716A, que es un antagonista del receptor CB1. Sin embargo, otros estudios experimentales provocando el cese del consumo de cannabinoides, han producido evidentes respuestas de abstinencia. En monos rhesus, el síndrome de abstinencia apareció al cesar de inyectar THC (Kaymcackalan S. 1973, Beardsley et al. 1986). Estos últimos datos, se han confirmado en diferentes estudios realizados en humanos, valorando que el cese de la administración de cigarrillos de marihuana o THC oral produce disminución del apetito, irritabilidad, cansancio, dificultades de sueño y falta de cooperación (Mendelson et al 1984, Jones et al.1976, Cohen et al. 1976). Budney et al. Indicaron que los síntomas de abstinencia en los estudios experimentales realizados en humanos eran menos severos que los que muestran estudios retrospectivos realizados en pacientes ambulatorios (Budney et al 1999). Una explicación de este hecho podría ser, que los realizados en pacientes ingresados a nivel experimental, no tienen en cuenta los estímulos ambientales que pueden condicionar algunos síntomas de abstinencia como el grupo de amigos, determinadas personas u objetos asociados con el consumo (Siegel S. 1983, Wikler 1980).

En cualquier caso, aunque no está claro cual es el significado clínico de estos síntomas de abstinencia, la aparición de los mismos, promueve la necesidad de estudios que evalúen su importancia (Budney et al 2001).

El objetivo de este estudio ha sido evaluar a través de un cuestionario en jóvenes multados por consumo de hachís el tipo de consumidores, las complicaciones y consecuencias derivadas del mismo y la aparición de síntomas de abstinencia al abandonar el consumo. Determinar si la frecuencia de consumo tiene relación con la aparición de síntomas, desmitificando de esa manera entre la población joven que el uso de cannabinoides no provoca síntomas ni tiene consecuencias físicas y/o psíquicas.

\section{MATERIAL Y METODOS}

145 pacientes (120 varones y 25 mujeres) de edades comprendidas entre los 15 a 32 años (Figura 1), edad media 20,6 años desviación típica (DT) $=3.23$, rellenaron un cuestionario anónimo(Anexo I) con el fin de evaluar los efectos producidos por el consumo y síntomas de abstinencia tras el cese del mismo.

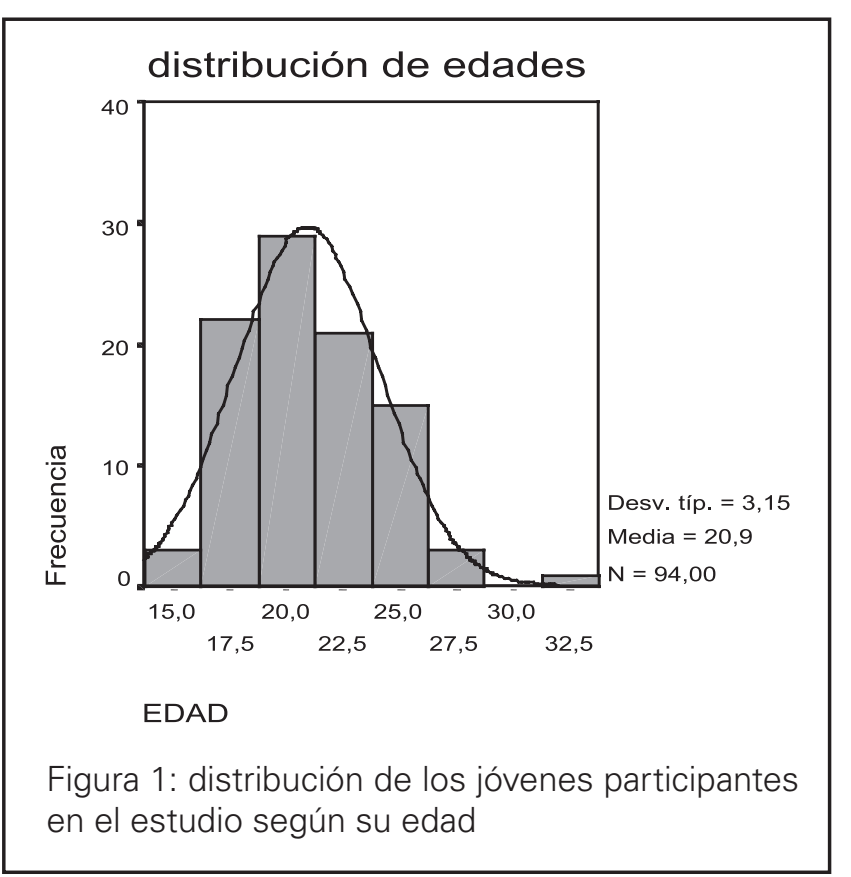

Los participantes acudieron al Programa Municipal de Drogas de Majadahonda, para realizar un programa específico de educación para la salud de tres meses de duración dirigido a jóvenes tras ser multados por consumo o tenencia de hachís para evitar el pago de la sanción administrativa de Mayo a Octubre de 2002.

Durante el programa se realizaron controles toxicológicos de orina para verificar la abstinencia de consumo de cannabis, teniendo en cuenta la liposolubilidad de la sustancia, que influye en el tiempo de desaparición en orina (vida media de 1-18 días), así como evaluar el consumo de otras sustancias. 
El cuestionario se dividió en 5 partes. La primera parte evalúa la edad del primer consumo y frecuencia en los últimos meses. Se diferenciaron dos tipos de consumidores en relación al patrón de consumo: los que fumaban todos los días y los que fumaban esporádicamente o fines de semana. Situación de consumo: cuando solían consumir, especificando diferentes situaciones como el consumo con amigos, en situaciones estresantes, estando solo, para ayudar a dormir o en cualquier situación. La segunda parte evalúa la historia de consumo de otras drogas, alcohol, tabaco drogas de síntesis, alucinógenos y cocaína valorando la edad del primer consumo, si consumían en el momento de rellenar el cuestionario y si el consumo era diario o los fines de semana.

La tercera parte se refiere a la aparición de síntomas de abstinencia tras la interrupción del consumo con un listado de 11 síntomas relacionados con el mismo. Se utilizó una escala validada denominada WDS (Withdrawal discomfort score) que pertenece a los 10 síntomas de aparición más habitual del cuestionario MWC (Marihuana Withdrawal Checklist) ( Budney et al 1999). Los síntomas medidos fueron irritabilidad, nerviosismo, inquietud, humor depresivo, enfado, dificultades para dormir, pesadillas, alteraciones en el apetito, cefaleas, aumento del consumo de tabaco. En nuestro cuestionario además se añadió otro síntoma, "deseo de consumo" que en otros estudios lo evalúan con otras escalas aparte que miden la intención y el deseo de consumo.

La cuarta parte del cuestionario valora las consecuencias derivadas del abuso de cannabis en relación con dificultades en obligaciones habituales (estudiar, trabajar, acudir a clase), si continua consumiendo hachís a pesar del peligro físico que implica hacerlo (conducir, trabajar con alguna maquinaria, deportes de riesgo) y si continúan consumiendo a pesar de tener problemas legales derivados del consumo.

La quinta y última parte evalúa las complicaciones agudas relacionadas con el consumo de cannabis midiendo 8 síntomas específicos, y otras complicaciones derivadas del consumo continuado como problemas con la familia, en los estudios o no tener ganas de hacer nada.

Análisis estadístico- los datos se expresan en \%. Para la comparación de variables categóricas se utilizó el análisis de proporciones en tablas $2 \times 2$ con la prueba $X^{2}$ normal o $X^{2}$ corregida por Yates en el caso de frecuencias esperada $<5$. Se considera significativo valores $\leq 0.05$.

La representación gráfica de las variables categóricas que contienen en general presencia o ausencia de distintos factores de riesgo, se representan en figuras de barras de frecuencias.

\section{RESULTADOS}

En el perfil de la muestra hay que destacar que el $98.6 \%$ estaban solteros, conviviendo con sus familias en el $96.6 \%$ de los casos, estudiantes en su gran mayoría $60.7 \%$, estudiando y trabajando el $23 \%$, trabajando el $12.4 \%$ y sin actividad ninguna el $4 \%$.

En cuanto al consumo de otras drogas el $49 \%$ del total de la muestra consumían alcohol en el momento de rellenar el cuestionario, siendo la edad del primer consumo los 15,4 años y el $48 \%$ fumaban tabaco siendo la edad del primer consumo a los 14.5 años. En relación al consumo de cocaína aunque solo contestaron 43 encuestados el $8 \%$ de los que contestaron consumían. Sólo el $2 \%$ consumían alucinógenos de los 49 que contestaron y en cuanto a drogas de síntesis de los 47 que contestaron, el primer consumo fue a los 18 años y solo el 5\% consumían ocasionalmente.

Todos los participantes consumían hachís, el 51.9 a diario, y el $48.1 \%$ esporádicamente o fines de semana. (Figura 2). La edad de primer consumo de cannabis fue a los 15,8 años (DT 1.86).

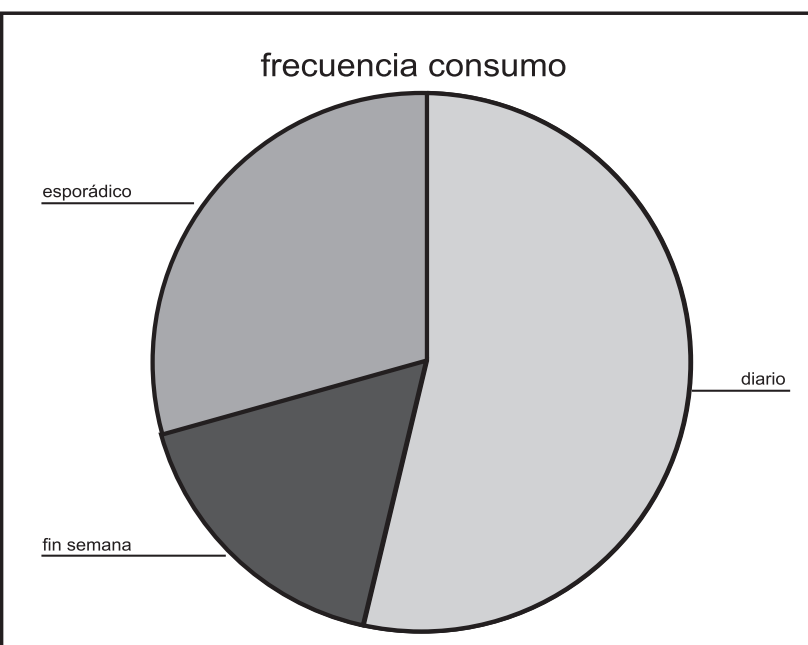

Figura 2. Distribución del consumo de cannabis, el $51.9 \%$ lo hace a diario, y el $48.1 \%$ esporádicamente o durante los fines de semana.

La gran mayoría consumían con amigos $67,1 \%$, aunque conviene resaltar que el $23 \%$ consumían en cualquier situación y el $18 \%$ contestaban que consumían cuando estaban solos, un $13 \%$ para que les ayudara a dormir y un $6 \%$ en situaciones estresantes.

Los resultados en relación a la pregunta sobre la aparición de síntomas de abstinencia al abandonar el consumo contestaron 133 de los encuestados, valorando algunas diferencias con significación estadística entre los dos tipos de consumidores analizados, siendo en el de consumidores habituales o diarios donde aparecieron diferencias en el deseo de consumo en relación a los de fin de semana o esporádico $\left(X^{2}=\right.$ 


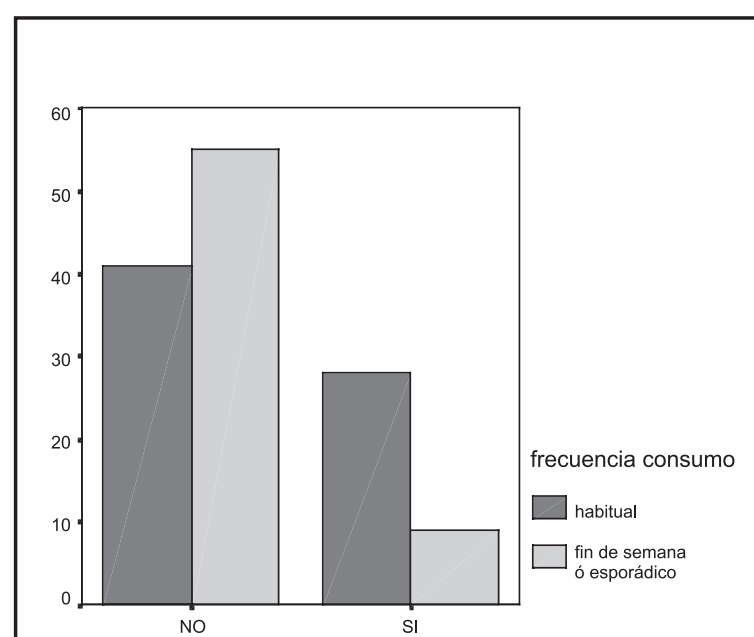

inquietud

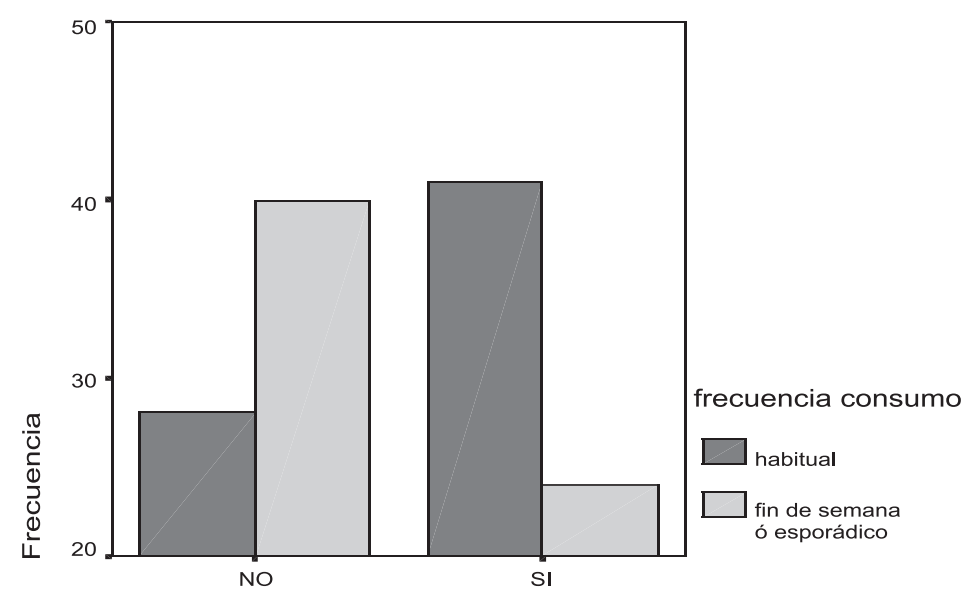

Deseos de consumir

Figura 3. Síntomas significativos tras el síndrome de abstinencia producido al interrumpir el consumo

$6.38, p<0.015)$ y síntomas de inquietud $p<0.0001$ mayor entre los consumidores habituales (Figura 3).

El resto de los síntomas de abstinencia medidos como nerviosismo, depresión o tristeza, enfado, somnolencia, alteraciones del sueño, pesadillas, alteraciones del apetito y aumento del consumo de tabaco, si bien existían diferencias entre ambos grupos no tuvie- ron significación estadística entre los consumidores habituales y los esporádicos. Aun así es necesario destacar el aumento del consumo de tabaco en el $49 \%$ de los casos, inquietud en el $27.8 \%$, alteraciones en el apetito en el $24 \%$ y síntomas depresivos en el 10.5\%. (Tabla 1).

Tabla 1 : valoración del síndrome de abstinencia producido por el consumo de hachís. Se consideraron significativos valores de $p<0,05^{*}$.

\section{Tras interrumpir de forma brusca en consumo ha sentido...?}

\begin{tabular}{llllll}
\hline $\begin{array}{l}\text { Síntomas } \\
\text { (n' total 133) }\end{array}$ & \% total SI & \% habitual & $\begin{array}{l}\text { \% F.semana } \\
\text { ó esporádico }\end{array}$ & $\begin{array}{l}\text { Chi- } \\
\text { cuadrado }\end{array}$ & Significación \\
\hline Deseos consumir & 48.9 & 30.8 & 18 & 6.38 & $\mathbf{0 . 0 1 5 *}$ \\
\hline irritabilidad & 13.5 & 9.8 & 3.8 & 3.451 & 0.078 \\
\hline nerviosismo & 21.1 & 14.3 & 6.8 & 3.62 & 0.057 \\
\hline inquietud & 27.8 & 21.1 & 6.8 & 11.6 & $\mathbf{0 . 0 0 0 1 *}$ \\
\hline Depresión ó tristeza & 10.5 & 6.8 & 3.8 & 0.96 & 0.403 \\
\hline Enfado & 9.8 & 6 & 3.8 & 0.538 & 0.565 \\
\hline somnolencia & 9.8 & 6 & 3.8 & 0.538 & 0.565 \\
\hline pesadillas & 5.3 & 3 & 2.3 & 0.08 & 1 \\
\hline Alteración apetito & 24.1 & 3 & 0 & 3.8 & 0.12 \\
\hline Aumento consumo tabaco & 48.9 & 27.8 & 21.1 & 1.298 & 0.255 \\
\hline
\end{tabular}

El abuso de cannabis provocó dificultades en el cumplimiento de las obligaciones habituales en el $25 \%$ de los encuestados siendo del $16.7 \%$ en los consumidores habituales y $8.3 \%$ de los de consumo esporádico sin significación entre ambos grupos $\left(X^{2}=2.2, p\right.$ $=0.138$ ). En cambio si se ha visto una clara diferencia entre ambos grupos en la pregunta sobre si han consumido en situaciones de peligro (conducir, manejar maquinas......), el $19.2 \%$ de los consumidores habituales lo han hecho frente al $3.3 \%$ de los que consumen esporádicamente $\left(X^{2}=12.82, p=0.0001\right)$ (Figura 4), siendo por último similar las respuestas entre ambos grupos en relación a la continuación del consumo a pesar de tener problemas asociados al consumo $20 \mathrm{y}$ $11 \%$ respectivamente. (Tabla 2 ). 
Tabla 2, valoración de los síntomas producidos por el abuso de cannabis. Se consideran resultados significativos valores de $p<0,05^{*}$

\begin{tabular}{|c|c|c|c|c|c|}
\hline $\begin{array}{l}\text { Síntomas } \\
\left(\mathrm{n}^{\circ} \text { total } 120\right)\end{array}$ & $\%$ total SI & $\%$ habitual & $\begin{array}{l}\text { \% F. semana } \\
\text { ó esporádico }\end{array}$ & $\begin{array}{l}\text { Chi- } \\
\text { cuadrado }\end{array}$ & Significación \\
\hline $\begin{array}{l}\text { dificultades en el cumplimiento de } \\
\text { las obligaciones habituales }\end{array}$ & 25 & 16.7 & 8.3 & 2.2 & 0.138 \\
\hline Consumo en situaciones de peligro & 22.5 & 19.2 & 3.3 & 12.82 & $0.0001 *$ \\
\hline $\begin{array}{l}\text { Continuación de consumo a pesar } \\
\text { de tener problemas sociales } \\
\text { asociados al consumo }\end{array}$ & 31.7 & 20 & 11.7 & 1.49 & 0.221 \\
\hline
\end{tabular}

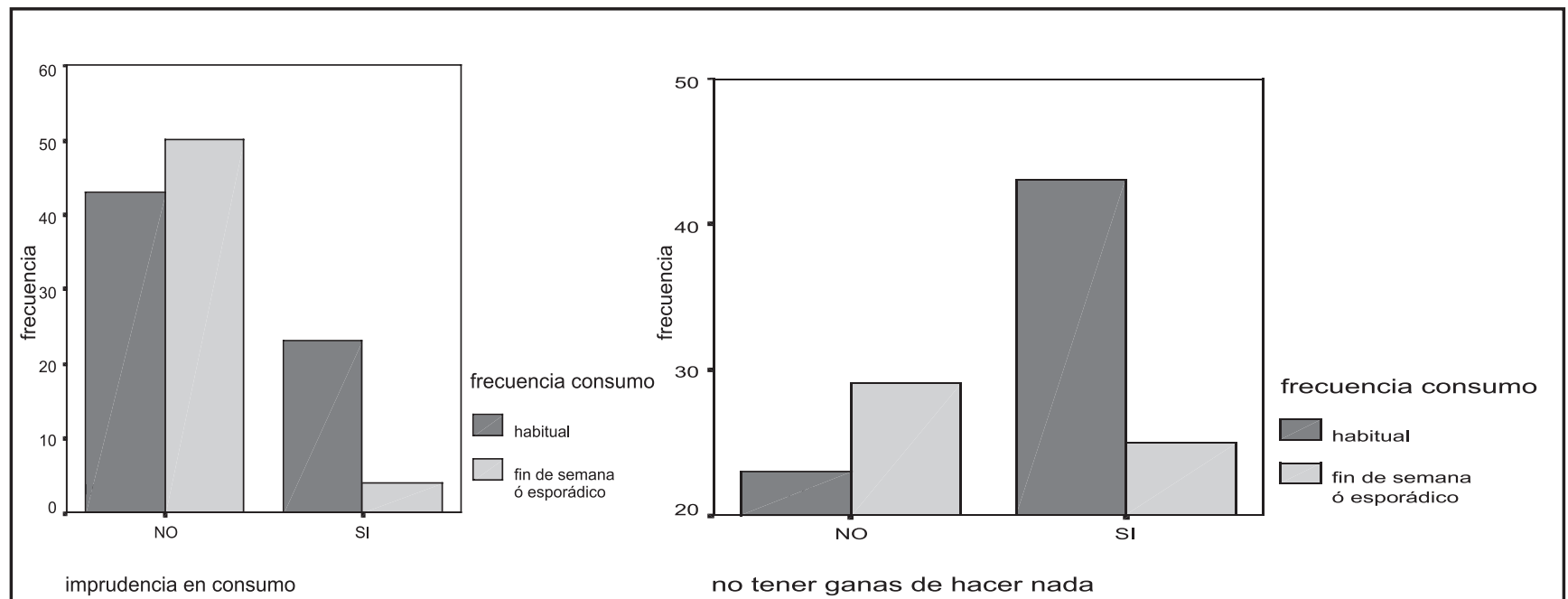

Figura 4, complicaciones más significativas producidas por abuso de cannabis, según la frecuencia del consumo

En el grupo de preguntas sobre complicaciones relacionadas con el consumo de hachís, no se observaron diferencias con valor significativo entre el grupo de consumidores habituales y ocasionales. Sin embargo es importante destacar la aparición de lipotimias en el $74.5 \%$ de los consumidores mayor en el grupo de consumo habitual $45.7 \%$ frente al $28.7 \%$ en el grupo de consumo esporádico, seguido de la aparición de reacciones paranoides en algún momento bajo los efectos del consumo en el $31.1 \%$ de los casos, arritmias en un $26 \%$ y alucinaciones en el $25 \%$ (Tabla 3 ).

Tabla 3: análisis de las complicaciones agudas producidas por abuso de hachís, se considera significativos aquellos síntomas con $\mathrm{p}<0,05$. Entre paréntesis se representa el número de jóvenes que contestaron a cada pregunta sobre el total de encuestados.

\begin{tabular}{llllll}
\hline $\begin{array}{l}\text { Síntomas } \\
\text { (n' total) }\end{array}$ & \% total SI & \% habitual & $\begin{array}{l}\text { \% F.semana } \\
\text { ó esporádico }\end{array}$ & $\begin{array}{l}\text { Chi- } \\
\text { cuadrado }\end{array}$ & Significación \\
\hline Lipotimias (94) & 74.5 & 45.7 & 28.7 & 1.77 & 0.182 \\
\hline Crisis de pánico (52) & 11.5 & 7.7 & 3.8 & 0.224 & 1 \\
\hline Depresión (55) & 16.4 & 14.5 & 1.8 & 4.17 & 0.064 \\
\hline Paranoia (61) & 31.1 & 18.0 & 13.1 & 0.014 & 0.905 \\
\hline Alucinaciones (60) & 25 & 16.7 & 8.3 & 3.7 & 0.762 \\
\hline Agresividad (54) & 13 & 11.1 & 1.9 & 2.33 & 0.22 \\
\hline Arritmia (58) & 25.9 & 17.2 & 8.6 & 0.540 & 0.552 \\
\hline
\end{tabular}


En cuanto a otro tipo de complicaciones relacionadas con el consumo como son los problemas familiares, en los estudios o no tener ganas de hacer nada, el $56.7 \%$ de 120 que contestaron, indicaron no tener ganas de hacer nada durante el tiempo que consumían, siendo claramente mayor en el grupo con consumo habitual 35.8\% en relación al grupo de consumo esporádico o de fin de semana 20.8\% (X2=4.3, $p=0.038$ ) (Figura 4). El 23.3\% tuvieron problemas con la familia sin relación con la cantidad de consumo y el $22.5 \%$ en los estudios. (Tabla 4 ).

Tabla 4: análisis de las complicaciones subagudas-crónicas producidas por abuso de hachís, se considera significativos valores de $p<0,05 *$. Entre paréntesis se representa el número de jóvenes que contestaron a cada pregunta sobre el total de encuestados.

\begin{tabular}{llllll}
\hline $\begin{array}{l}\text { Síntomas } \\
\text { (n total) }\end{array}$ & \% total SI & \% habitual & $\begin{array}{l}\text { \% F.semana ó } \\
\text { esporádico }\end{array}$ & $\begin{array}{l}\text { Chi } \\
\text { cuadrado }\end{array}$ & Significación \\
\hline Problemas familiares (120) & 23.3 & 12.5 & 10.8 & 0.03 & 0.862 \\
\hline Problemas en los estudios (120) & 22.5 & 13.3 & 9.2 & 0.255 & 0.613 \\
\hline No tener ganas de hacer nada (120) & 56.7 & 35.8 & 20.8 & 4.3 & $\mathbf{0 . 0 3 8 *}$ \\
\hline
\end{tabular}

\section{DISCUSIÓN}

Los resultados del cuestionario realizado en jóvenes consumidores de cannabis reflejan que el patrón más habitual de consumo es asociado al tabaco y alcohol. El $49 \%$ del total de la muestra de nuestro estudio consumían alcohol en el momento de rellenar el cuestionario, siendo la edad del primer consumo los 15,4 años y el $48 \%$ fumaban tabaco siendo la media de edad del primer consumo los 14.5 años. La existencia de interacciones entre el THC con varias drogas de abuso ha sido demostrada en animales de experimentación y en humanos. Estas interacciones como aparecen recogidas en la revisión realizada por Ramos et al. están relacionadas con las dosis y presentan variaciones en relación con las diferencias existentes entre sus consumidores en cuanto a la sensibilidad de la droga o a su absorción, almacenamiento, metabolismo, y eliminación (Ramos et al 2001). Se ha propuesto la existencia de un mecanismo común de reforzamiento positivo producido por las drogas de abuso en el que entre otros estarían implicados los sistemas dopaminérgico, opioide y gabaérgico (Koob y Le Moal ,1997). La actuación sobre estos sistemas de cualquier droga de abuso podría afectar la actividad de los cannabinoides, a la vez que éstos podrían influir sobre la actuación de todas ellas. La mezcla de cannabis y alcohol produce interacciones de tal manera que el consumo de alcohol aumenta los efectos clínicos del THC (Doty et al 1992). A su vez fumar marihuana aumentó alguno de los efectos del etanol como somnolencia, taquicardia y el desacoplamiento de determinadas actividades del sistema nervioso central entre las que se encuentran la percepción, el conocimiento y la función motora. (Ramos et al 2001). Los efectos del cannabis sobre el metabolismo del etanol son escasos, por lo que su actuación parece estar relacionada con la disminución de los niveles de este alcohol en sangre (Lukas et al
1992). Aunque no se conoce todavía el mecanismo utilizado por el etanol para modificar el sistema cannabinoide endógeno, si se sabe que el etanol inhibe la síntesis de anandamida Datos experimentales indican que el etanol activa algunas de las acetiltranferasas que participan en la formación de los lípidos presentes en las membranas cerebrales. Además la exposición crónica facilita la entrada de calcio en neuronas (Zhen et al 1998). De esta forma el etanol podría modular la síntesis de anandamida por la activación directa de ambas enzimas (Ramos J.A et al. 2001). Los resultados de otros muchos estudios (Calafat et al 1989, Comas 1990, Elzo et al 1992, ;PNSD 1994, 1996, 2000), confirman que las drogas de comercio legal (tabaco y alcohol) son las que más se consumen. Además los datos de estudios realizados en 1995, 1996 y 2000 por el Plan Nacional sobre Drogas reflejan que nos encontramos ante una situación en la que el monoconsumo es prácticamente inexistente y prevalece una pauta de uso de varias sustancias. Se asocia, en primer lugar, el alcohol, tabaco y cannabis (Campins 1996, PND 1995, PND 2000). Una investigación sobre el consumo de drogas en adolescentes en una población joven realizado por Graña et al. indica que el consumo múltiple de tabaco, alcohol y cannabis incrementa la toxicidad de las mismas, dificultando las intervenciones y empeorando las consecuencias sociales, personales y de salud (Graña et al. 2000). Los resultados de un estudio realizado en 3204 jóvenes en Alemania indican que el consumo de cannabis es la sustancia de uso más frecuente entre jóvenes, seguida de estimulantes y alucinógenos, incrementándose el poliabuso entre jóvenes de 14 a 24 años y valoran que un tercio de ellos comienzan a consumir antes de los 16 años, aumentando durante la adolescencia y la juventud (Perkonigg A. et al., 1998). Sin embargo nuestros resultados en relación al consumo de otras sustancias como alucinógenos, 
drogas de síntesis y cocaína, son poco relevantes, aunque hay que destacar que el número de jóvenes que contestaron estas preguntas fue muy reducido. Aunque una de las formas mas extendidas de consumo de hachís es fumándola mezclada con tabaco, no se han realizado aún los estudios oportunos sobre las posibles interacciones entre el THC y la nicotina (Ramos et al, 2001)

Los resultados del cuestionario confirman la aparición de síntomas de abstinencia, con suficiente relevancia ya que fueron reflejados en las respuestas de los jóvenes en este estudio, destacando el aumento del consumo de tabaco, inquietud, alteraciones en el apetito y síntomas depresivos, aunque sin diferencias significativas entre el grupo de consumidores habituales y el de fin de semana.

Estos hallazgos también aparecen en otros estudios realizados en población general (Haney et al 1999, Crowley et al 1998, Cottler et al 1995, Weisbeck et al 1996), en los que aparecen síntomas de abstinencia al interrumpir el consumo de cannabis. El realizado recientemente en pacientes ambulatorios por Kouri y Pope en pacientes que fumaban marihuana diariamente, mostró aumento de ansiedad, irritabilidad, alteraciones de humor y disminución del apetito durante los 28 días posteriores al consumo, aunque los síntomas referidos como en el caso de nuestro este estudio, fueron difíciles de evaluar ya que eran datos retrospectivos que referían los pacientes (Kouri EM y Pope HG 2000).

Otros resultados del estudio realizado, son las diferencias con significación estadística entre los que consumían diariamente en relación a los que consumían los fines de semana en dos síntomas de abstinencia , "deseo de consumo" y "síntomas de inquietud". Algunos autores han descrito la aparición de un síndrome de abstinencia moderado en consumidores habituales de preparaciones de cannabis con elevadas concentraciones de principios activos (Hollister, 1986; Haney et al. 1997; Kouri et al 1999, 2000). Los signos más prominentes fueron irritabilidad, inquietud, anorexia, ansiedad y aumento de la vigilia (Haney et al. 1999, Kouri et al 1999, 2000). Sin embargo, el síndrome de abstinencia no fue lo suficientemente severo como para alterar significativamente la vida cotidiana del individuo (Kouri et al 2000). La severidad de la abstinencia dependió de las dosis de THC, la frecuencia de consumo y la duración del uso de la sustancia. El desarrollo de dependencia de cannabinoides inducida por la administración crónica de THC, también ha sido estudiado en voluntarios sanos (Jones et al 1981) donde los síntomas descritos fueron similares a los observados en grandes consumidores de cannabis (Maldonado R. 2002). Estudios realizados en animales de experimentación confirman la existencia de una relación directa entre la dosis de THC y la severidad de la abstinencia. Budney et al. realizaron un estudio experimental con 12 consumidores diarios de marihuana durante 16 días consecutivos. Los primeros 5 días fumaban habitualmente, se interrumpía el consumo los días 6-8, volvían a consumir los días 9-13 y de nuevo estaban en abstinencia los días 14 a 16. Se observó que los días de no consumo aparecieron algunos síntomas de abstinencia, como deseo de consumo, disminución del apetito, dificultades para dormir y pérdida de peso. El resto de síntomas de abstinencia sólo aparecieron durante una de las fases de la abstinencia (agresividad, irritabilidad, pesadillas, falta de descanso) (Budney et al 2001).

Los dos patrones de consumo observados en nuestro estudio, diario y fines de semana, si bien nos acercan a una frecuencia de consumo habitual o crónica y fines de semana o aguda, tienen el inconveniente de no ofrecer una medición de las dosis de cannabis que permita diferenciar la aparición de determinados síntomas, en relación con la cantidad de consumo previo, la severidad de los mismos y la posible aparición de dependencia.

En cuanto a los síntomas provocados por abuso de cannabis hay que destacar que el uso crónico de marihuana se ha asociado con un estado caracterizado por apatía y pérdida de motivación con deterioro en los rendimientos académicos y cambios en la conducta que algunos autores han descrito con el término de síndrome amotivacional (Pope et al 1996, Smith 1968). Aunque la explicación y los mecanismos de dicha asociación no está bien establecida, parece que los pacientes que lo presentan muestran una serie de rasgos previos similares a los que se describen como propios de este síndrome, es decir que el cannabis provocaría un aumento en la intensidad de dichos rasgos (Nuñez L.A 2002).Los síntomas característicos de este síndrome serian apatía, disminución de la productividad, déficit generales e interacción social disminuida (Sharma, 1975). En nuestro estudio hay que destacar que el consumo de cannabis provocó una sintomatología específica de "no tener ganas de hacer nada" en el $56.7 \%$ de los encuestados, siendo claramente mayor en el grupo con consumo habitual, en relación al grupo de consumo esporádico o de fin de semana. La valoración de si este síntoma apareció mas en aquellos con rasgos previos no es posible al ser un cuestionario anónimo, aunque si quedó claro que la frecuencia de consumo influyó en su aparición.

En cambio si se observó una clara diferencia entre ambos grupos en la pregunta sobre si habían consumido en situaciones de peligro como conducir, 19.2\% de los consumidores habituales lo habían hecho frente al 3.3\% de los que consumían esporádicamente. De acuerdo con la bibliografía en experimentación 
animal (Dewy, 1986), encontramos un efecto diferencial del THC sobre la conducta espontánea de la rata en el sentido de que el efecto de dosis bajas sería de tipo estimulador (aumento de las conductas motoras) y dosis altas ( por encima de 1,6 mg/kg de 9-THC) tendrían un efecto de tipo depresor (disminución de dichas conductas) y este efecto se atenuaría en los tratamientos más crónicos, debido al desarrollo de un fenómeno de tolerancia ( Palomo et al. 2001). Existen evidencias a favor de que el sistema cannabinoide participa en el control del movimiento como lo hacen otros neurotransmisores mas clásicos como la dopamina, GABA o el glutamato (Consroe, 1998; Sañudo-Peña et al 1999; Fernández Ruiz et al 2002). La actividad endocannabinoide juega un papel importante a nivel modulador en la funcionalidad de los ganglios basales, con datos bioquímicos y farmacológicos que apoyan esta función, sentando las bases para explicar el por qué aquellas sustancias que son activas sobre las diferentes proteínas que forman parte del sistema endocannabinoide (receptores, transportador, enzimas), pueden tener un efecto beneficioso en el tratamiento de la disfunción motora en enfermedades extrapiramidales, o en patologías no motoras que presentan síntomas extrapiramidales. Sin embargo, este posible efecto terapéutico en enfermedades con sintomatología extrapiramidal, no es aplicable al efecto hipoquinético en el que coinciden la gran mayoría de los autores que produce la activación de los receptores CB1 y que en el caso de conducir bajo sus efectos o manejar maquinaria, puede tener repercusiones negativas aumentando la peligrosidad (Lastres-Becker et al 2002) como ocurre en los jóvenes de este estudio.

Entre las complicaciones aparecidas bajo los efectos del hachís, aunque no todos los encuestados contestaron, es importante destacar que el $74.5 \%$ de los 94 que contestaron tuvieron lipotimias y el $31.1 \%$ de 61 cuestionarios contestados tuvieron reacciones paranoides, siendo llamativo otras reacciones como alucinaciones $(25 \%)$, cuadros depresivos (16.4\%), agresividad (13\%)y crisis de pánico en un $11.5 \%$. No existe un cuadro específico definido en relación a los trastornos psíquicos relacionados con el consumo de cannabinoides, siendo muy variables dependiendo de la predisposición individual y del contexto. Se pueden producir estados de ansiedad aguda que puede llegar al pánico, o a la disociación histérica, episodios maniformes o ideación suicida (Palomo et al. 2001). También se puede producir una reacción aguda paranoide o esquizofreniforme (Mathers y Ghodes ,1992) en sujetos predispuestos, reacciones agudas orgánicas tales como desorientación, alteración de la memoria, confusión, delirios, alucinaciones y síntomas paranoides (Talbott y Teague 1996). No existe una conclusión definitiva acerca de si estos síntomas son causados directamente por el cannabis ( Spencer
,1970), ni tampoco si el cannabis precipita la enfermedad o estaba ya enfermo.

\section{CONCLUSIONES}

Los resultados obtenidos en este cuestionario de una muestra de población joven consumidora de hachís, aportan datos que evidencian una tendencia clara al policonsumo de alcohol, tabaco y cannabis con el incremento de riesgos que conlleva, abriendo un abanico de interacciones fisiológicas y farmacológicas del cannabis con otras drogas de abuso. También proporciona información sobre la aparición de complicaciones vinculadas al consumo, relacionando algunas de ellas con los dos patrones de consumo evaluados, diario y fines de semana y en otros casos sin relación con el tipo de consumo. En este sentido, la falta de motivación provocada en el grupo de consumidores habituales, determina consecuencias en la realización de actividades cotidianas como estudiar o asistir a clase, así como otros riesgos al continuar conduciendo vehículos y manejando maquinaria bajo sus efectos.

Confirma la aparición de síntomas de abstinencia al abandonar el consumo, destacando algunas diferencias entre los dos patrones de consumo analizados, diario y esporádico o de fin de semana en algunos síntomas específicos, lo que nos lleva a reflexionar sobre el grado de tolerancia que se adquiere con el tipo de consumo y la posible dependencia a cannabinoides.

Quedan muchas dudas por resolver en relación al consumo de cannabis en jóvenes, las complicaciones derivadas del mismo en relación al tipo de consumo, el poliabuso de drogas y la aparición de sintomatología de abstinencia. No queda claro la cantidad necesaria que determina la aparición de esta sintomatología ni tampoco se ha podido analizar en los resultados de este cuestionario anónimo factores de vulnerabilidad individual que predispongan la aparición de determinados estados emocionales reactivos.

Serán necesarios muchos mas estudios clínicos entre esta población que nos permita avanzar en este camino.

\section{REFERENCIAS}

Abood ME, Martin BR, (1992), Neurobiology of marijuana abuse, Trends Pharmacology Science, 13:201-207.

Aceto MD, Scates SM, Lowe JA y Martin BP, (2001), Spontaneous and precipitated withdrawal with a synthetic cannabinoid, WIN 55,212-2,European Journal of Pharmacology,416:75-81. 
American Psychiatric Association, (1994), Diagnostic and Statistical Manual of Mental Disorders, Fourth Edition, Washington,DC, American Psychiatric Association.

Beardsley PM, Balster RL, Harris SL, (1986), Dependence on tetrahydrocannabinol in rhesus monkeys, Journal Pharmacology Experimental Therapy, 239: 311-319.

Budney AJ, Novy PL, Hughes JR, (1999), Marijuana withdrawal among adults seeking treatment for marijuana dependence, Addiction, 94: 1311-1322.

Budney AJ, Higgins ST, Radonovich KJ, Novy PL, ( 2000), Adding voucher-based incentives to coping skills and motivational enhancement improves outcomes during treatment for marijuana dependence, Journal Consulted Clinical Psychopharmacology, 68 :10511061.

Budney AJ, Jughes J, Moore B, Novy PL, (2001), Marijuana abstinence effects in marijuana smokers maintained in their home environment, Archais General of Psychiatry, 58:917-924 American Medical Association.

Campins M, Gasch J, Hereu P, Roselló J, Vaqué J, (1996), Consumo y actitudes de los adolescentes frente a sustancias adictivas: encuesta de prevalencia, Anales de pediatría, 45(5),475-478.

Calafat A, Amengual M, Megias G, Borrás M, (1989), Consumo de drogas en enseñanza media, Comparación entre 1981-1988, Revista española de drogodependencias, 14: 9-28.

Cohen S, Lessin PJ, Hahn PM, Tyrell ED (1976), A 94 day cannabis study, In: Braude MC, Szara S,eds, Pharmacology of Marihuana, New York, NY: Raven Press, 621-626.

Comas D, (1990), El síndrome de haddhock: alcohol y drogas en las enseñanzas medias, Madrid: CIDE.

Consroe P, (1998) Brain cannabinoid systems as targets for the therapy of neurological disorders, Neurobiology Disease, 5: 534-551.

Crowley TJ, Mcdonald MJ, Whitmore EA, Mickulich SK, (1998), Cannabis dependence withdrawal and reinforcing effects among adolescents with conduct symptoms and substance use disorders, Drug and Alcohol Dependence, 50: 27-37.

Cottler LB,Schuckit MA,Helzer JE, Crowley T, Woody G, Nathan P, Hughes J,(1995), The DSM IV field trial for substance use disorders: major results, Drug and Alcohol Dependence, 38:59-69.

Dewey WL, (1986), Cannabinoid pharmacology, Pharmacological Review 38(4):151-178.

Doty P, Dykstra LA, Picker MJ, (1992), Delta-9tetrahydrocannabinol interactions with phencyclidine and etanol, Effects on accuracy and rate of responding, Pharmacology, Biochemical Behaviour, 43,61-70.

Elzo J,(1992), Drogas y Escuela IV, BUP-FP, Donosita-San Sebastián: Escuela Universitaria de Trabajo Social.

Fernández-Ruiz JJ, Lastres-Becker I, Cabranes A, González S y Ramos JA, (2002) Endocannabinoids and basal ganglio functionality, Prostaglandins Leuko, Essent, Fatty Acids, 66: 623-273.
Graña J L, Muñoz M,J, Delgado S, (2000), Investigación sobre el consumo de drogas en adolescentes de Majadahonda: Factores de riesgo y protección, Excmo, Ayto, de Majadahonda con la colaboración de la Agencia Antidroga de la Comunidad de Madrid. I.S.B.N: 84-922204-4-4-9.

Haney M, Ward AS, Comer SD, Wards As, Foltin RW, Fischman MW, (1997), Factors incluencing marijuana self-administration by humans, Behaviour Pharmacology, 8: 101-112.

Haney M, Comer SD, Ward AS, Foltin RW, Fischman MW, (1999), Abstinence symptoms following oral THC administration to humans, Psychopharmacology 14 : 385-394.

Haney M, Comer SD, Ward AS, Foltin RW,Fischman MW, (1999), Abstinence symptoms following smoked marijuana in humans, Psychopharmacology 14: 395404.

Hollister LE, (1986), Health aspects of cannabis, Pharmacology Reviews, 38:1-20.

Jones RT, Benowitz N,(1976), The 30 day trip: Clinical studies of cannabis tolerance and dependence, In: Braude MC, Szara S, eds, Pharmacology of Marihuana New York, NY: Raven Press, 627- 642.

Jones RT, Benowitz NL y Herning RI, (1981), Clinical relevance of cannabis tolerance and dependence, Journal Clinical Pharmacology,21: 143S-152S.

Kaymcackalan S, (1973), Tolerance to and dependence on cannabis, Bull, Narcotic,25:39-47.

Koob y Le Moal, (1997), Dug Abuse:hedonic homeostatic dysregulation, Science, 278:52-58.

Kouri EM, Pope HG, (2000), Abstinence symptoms during withdrawal from chronic marijuana use, Experimental Clinical Psychopharmacology 8:483-492.

Kouri EM, Pope HG, (2000), Abstinence symptoms during withdrawal from chronic marijuana use, Experimental Clinical Psychopharmacology, 8:483-492.

Lukas S, Benedict R, Mendelsohn JH, Kouri E, Scholar M, Amass L, (1992), Marijuana attenuates the rise in plasma etanol level in human subjects, Neuropsychopharmacology, 7: 77-81.

Lastres-Becker, Cabranes A, De Lago E, Fernandez Ruiz J, (2002), Cannabinoides y actividad motora, En: Guia Básica sobre los Cannabinoides, (Sociedad Española de Investigación sobre Cannabinoides), Madrid,83-95.

Maldonado R, (2002), Tolerancia y dependencia a cannabinoides, En: Guia Básica sobre los Cannabinoides, (Sociedad Española de Investigación sobre Cannabinoides), Madrid, 144-152.

Mathers DC, Ghodse AH (1992), Cannabis and psychotic illness, British Journal of Psychiatry, 161: 648-53

Meana J y Ulibarri I,( 1999),Acciones farmacológicas y utilidad terapéutica de los derivados del cannabis, En: Cannabis ¡Hasta donde!, (Cabrera J, ed,) Agencia Antidroga,61-76.

Mendelson Jh, Mello NK, Lex BW, Bavli S,(1984), Marijuana withdrawal syndrome in a woman, American Journal of Psychiatry, 141: 1289-1290. 
Nahas GC, (1984), Toxicology and pharmacology, En: Marihuana in Science and Medicine (Nahas GG, Harvey DJ, Paris M y Brill ,eds) Raven Press New Cork pp, 109-246.

Nuñez Dominguez L,A (2002), Efectos psicológicos de los cannabinoides, En: Guia Básica sobre los Cannabinoides, (Sociedad Española de Investigación sobre Cannabinoides), Madrid, 144-152.

Palomo T, Gorriti MA, Ponce G, Archer T, Jiménez Arriero MA, (2001), De la investigación básica sobre los cannabinoides al estudio de las alteraciones clínicas asociadas a su consumo, En: Avances Neurocientíficos y Realidad Clínica III, Trastornos adictivos, (Palomo T, Beninger RJ, Jiménez-Arriero MA, Archer T,eds), Fundación Cerebro y Mente, Editorial Síntesis,Madrid, pp, 197-223.

Perkonnig A, Lieb R, Hofler M, Schuster P, Sonntag $H$, Witchen $H, U$ (1999), Pattern of cannabis use, abuse and dependence over time: incidence, progression stability in a sample of 1228 adolescents, Addiction, 94: 1663-1678.

Pope HG y Jurgelun Todd D, (1996), The residual cognitive effects of heavy marijuana use in college students, JAMA,275 (7)521-527.

Plan Nacional sobre Drogas, (1994), Encuesta sobre drogas a la población escolar, Madrid: Delegación del Gobierno para el Plan Nacional sobre Drogas, Ministerio del Interior.

Plan Nacional sobre Drogas, (1996), Encuesta sobre drogas a la población escolar, (Informe preliminar), Madrid: Delegación del Gobierno para el Plan Nacional sobre Drogas, Ministerio del Interior.

Plan Nacional sobre Drogas (2000) Memoria 2000, Madrid, Delegación del Gobierno para el Plan Nacional sobre Drogas, Ministerio del Interior.

Ramos JA, González S, Cabranes A, Fernández-Ruiz J, (2001), Interacciones fisiológicas y farmacológicas del cannabis con otras drogas de abuso, En: Avances
Neurocientíficos y Realidad Clínica III, Trastornos adictivos, (PalomoT, Beninger RJ, Jiménez-Arriero MA, Archer T,I, eds,) Fundación Cerebro y Mente, Editorial Síntesis, Madrid, pp,225-235.

Sañudo-Peña MC, Tsou K, Walter JM, (1999), Motor actions of cannabinoids in the basal ganglio output nuclei, Life Science,65:703-713.

Siegel S, (1983), Classical conditioning drug tolerante and drug dependence, In: (Israel Y, Glaser FB, Kalant $H_{\text {, }}$ Popham RE, Schmidt W, Smart RG, eds,) Research Advances in Alcohol and Drug Problems, New York, NY, Plenum press, 1983:207-246.

Sharma BP, (1975), Cannabis and its users in Nepal, British Journal of Psychiatry 127:550-552.

Smith DE, (1968), Acute and chronic toxicity of marijuana, Journal Psychedel Drug, 2:37-47.

Smith NT, (2002), A review of th published literature into cannabis wihdrawal symptoms in human users, Addiction, 97(6): 621-632.

Spencer DJ,(1970), Cannabis induced psicosis, West Indian Medicine Journal 19(4):228-230.

Substance Abuse and mental Health Services Administration, (1999), Summary of finding from the 1998 Household Survey on Drug Abuse, Rockville, Md:US Dept of Health and Human Services.

Talbott JA y Teague JW,(1996), Marihuana psicosis, Acute toxic psicosis associated with the use of cannabis derivates, JAMA 210(2): 299-302.

Weisbeck GA, Schutkit MA, Kalmijn JA, Tipp JE, Bucholz KK, Smith TL, (1996), An evaluation of the history of a marijuana withdrawal syndrome in a large population, Addiction, 91: 1469-1478.

Wickler A, (1980), Opioid Dependence: Mechanism and Treatment, New York, NY: Plenum Press.

Zhen T, Li W, Zhang A, Altura BT y Altura BM, (1998), Alphatocopherol prevents ethanol-induced elevation of $\mathrm{Ca}++$ in cultured canine cerebral vascular smooth muscle cells, Neuroscience Letters 245:17-20. 


\section{ANEXO 1}

\section{ENCUESTA DE HACHÍS EN POBLACIÓN JOVEN DEL DISTRITO SANITARIO DE MAJADAHONDA}

\section{DATOS DE FILIACIÓN}

Fecha

$\mathrm{N}^{\circ}$ Historia

\section{DATOS SOCIODEMOGRÁFICOS}

Sexo

Fecha de Nacimiento Edad.

Estado Civil: (marque con una X)

Soltero
Casado
Separado
Viudo

Viudo

Convivencia: (marque con una X)

Familiar

Solo

Pareja

Lugar de Residencia

Municipio

Situación Laboral

Estudia ..si...no

Trabaja si...no

Estudias y trabajas....si...no

Sin actividad............si...no

Estudios: (marque con una $\mathrm{X}$ )

Sin estudios........

Primarios...........

Medios......

Universitarios....

\section{SÍNDROME DE ABSTINENCIA}

Señala con una cruz los síntomas de abstinencia si los has tenido alguna vez usando la escala adjunta.

(¿Tras interrumpir el consumo de forma brusca has sentido....?)

- deseo de consumo

- irritabilidad.

- nerviosismo

- inquietud....

- depresión o tristeza

- enfado.

- somnolencia

- pesadillas
- $\quad$ alteraciones en el apetito...........

- cefalea..

- aumento del consumo de tabaco.

Edad del primer consumo. .años

Frecuencia del consumo en los últimos meses: (señala con una $\mathrm{X}$ )

- Diario......

- Fines de semar

- Esporádico.

Cuando sueles consumir:

- $\quad$ Estando con amigos, bar, discoteca...........

- Situaciones estresantes.

- Cuando estás solo

- Para ayudarte a dormir.

- En cualquier situación.

\section{ABUSO DE CANNABIS}

El consumo de hachís te ocasiona dificultades en el cumplimiento de tus obligaciones habituales (estudiar, trabajar, acudir a clase........)

SI................NO

Has tenido problemas legales repetidos por el hachís SI................NO......

Consumes hachís en situaciones en las que hacerlo implica un peligro físico. Por ejemplo conducir, trabajar con alguna maquinaria, deportes de riesgo.

SI............. NO......

Continúas consumiendo a pesar de tener problemas sociales derivados de su uso

Sl..........NO ....

COMPLICACIONES AGUDAS POR CONSUMO DE CANNABIS ¿Has tenido alguna de estas reacciones tras el consumo?

- Lipotimias (amarillo) Si....No........... $\mathrm{N}^{\circ}$ Veces...........

- Crisis de pánico Si....No........... $\mathrm{N}^{\circ}$ Veces......

- Paranoia Si.....No........... $\mathrm{N}^{\circ}$ Veces............

- Desorientación Si....No.............. Veces.

- Alucinaciones $\mathrm{Si}$....No............ $\mathrm{N}^{\circ}$ Veces...........

- Agresividad Si.....No............... ${ }^{\circ}$ Veces....

\section{COMPLICACIONES SUBAGUDAS-CRÓNICAS}

Habitualmente fumar hachís ha dado lugar a:
- Problemas familiares
Si...................
- Problemas en estudios
Si...................
- No tener ganas de hacer nada (apatía) Si........No...... ...

HISTORIA DE CONSUMO DE OTRAS DROGAS

\begin{tabular}{|l|l|l|l|l|l|}
\hline & Alcohol & Tabaco & $\begin{array}{l}\text { Drogas } \\
\text { síntesis }\end{array}$ & Alucinógenos & Cocaína \\
\hline Edad primer comsumo & & & & & \\
\hline $\begin{array}{l}\text { Edad consumo. } \\
\text { Fines de semana }\end{array}$ & & & & & \\
\hline $\begin{array}{l}\text { Edad consumo } \\
\text { todos los dias }\end{array}$ & & & & & \\
\hline Consumo actual & & & & & \\
\hline
\end{tabular}


